\title{
Comparing Kirchhoff-approximation and boundary-element models for computing gadoid target strengths
}

\author{
Kenneth G. Foote ${ }^{a)}$ \\ Woods Hole Oceanographic Institution, Woods Hole, Massachusetts 02543 \\ David T. I. Francis \\ School of Electronic and Electrical Engineering, University of Birmingham, Edgbaston, Birmingham B15 2TT, \\ United Kingdom
}

(Received 16 April 2001; revised 4 December 2001; accepted 14 January 2002)

\begin{abstract}
To establish the validity of the boundary-element method (BEM) for modeling scattering by swimbladder-bearing fish, the BEM is exercised in several ways. In a computation of backscattering by a 50-mm-diam spherical void in sea water at the four frequencies 38.1, 49.6, 68.4, and 120.4 $\mathrm{kHz}$, agreement with the analytical solution is excellent. In computations of target strength as a function of tilt angle for each of 15 surface-adapted gadoids for which the swimbladders were earlier mapped, BEM results are in close agreement with Kirchhoff-approximation-model results at each of the same four frequencies. When averaged with respect to various tilt angle distributions and combined by regression analysis, the two models yield similar results. Comparisons with corresponding values derived from measured target strength functions of the same 15 gadoid specimens are fair, especially for the tilt angle distribution with the greatest standard deviation, namely $16^{\circ}$. (C) 2002 Acoustical Society of America. [DOI: 10.1121/1.1458939]
\end{abstract}

PACS numbers: 43.30.Gv, 43.30.Sf [DLB]

\section{INTRODUCTION}

Knowledge of fish target strength has long been recognized to be vitally important in acoustic measurements of fish density, witnessed by the bibliographies in Refs. 1-5. In the echo integration method, it appears through the backscattering cross section as a divisor of the area backscattering coefficient or like proportional quantity. ${ }^{6}$ In the echo counting method, it appears in the expression for the acoustic sampling volume. ${ }^{7}$

There is a multiplicity of methods to determine target strength. The 20 or so methods cited in a 1991 study $^{3}$ have since been augmented significantly by a number of new techniques, including both empirical methods ${ }^{8,9}$ and theoretical models, especially those based on the deformed-cylinder model $^{10}$ and boundary-element method. ${ }^{11}$ These have been accompanied by novel applications, for example, to salmon and trout, ${ }^{8,9}$ cod (Gadus morhua), ${ }^{12}$ orange roughy (Hoplostethus atlanticus), ${ }^{13}$ and pollack (Pollachius pollachius). ${ }^{14,15}$

Modeling fish target strength, in particular, offers opportunities of investigation that may otherwise be unrealizable or exceedingly cumbersome, as in the case of orange roughy, a fish that resides at $700-1500 \mathrm{~m}$ depth. ${ }^{16}$ At the same time, models generally have a domain of applicability that must be defined and respected in applications.

A number of distinct scattering models have been applied to fish. These have been based principally on simple shapes such as the sphere at low frequencies ${ }^{17-19}$ and prolate ellipsoid, ${ }^{20-22}$ or the actual shape ${ }^{14,15,23-26}$ called "mapping" method by McClatchie et al. ${ }^{25}$ Only the first two models have exact solutions in general, thus the matter of approxi-

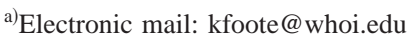

mation must be addressed, at least for realistic shapes at relatively high frequencies, where the wavelength is not very long compared to the size of significant scattering organs. $^{23,24}$

The deformed fluid-cylinder model ${ }^{10,27-29}$ has been very popular and has been the object of a major study. ${ }^{30}$ Essentially, it reduces an observed shape to a series of axisymmetric cylinders. The method has been realized by Clay and Horne for Atlantic cod, ${ }^{12}$ McClatchie and Ye for orange roughy, ${ }^{13} \mathrm{McClatchie}$ et al. for barracouta (Thyrsites atun), red cod (Pseudophycis bachus), and southern blue whiting (Micromesistius australis), ${ }^{25}$ and Sawada et al. for walleye pollock (Theragra chalcogramma). ${ }^{22}$ Excepting the cited case of Atlantic cod, each model has been based entirely on the swimbladder as a deformed cylinder. The swimbladder is important in the Atlantic cod model, but this includes other parts of the fish too, again represented as finite cylinders. ${ }^{12}$

The exact shape of the swimbladder has also been considered more directly in the Kirchhoff-approximation model for pollack and saithe (Pollachius virens), ${ }^{23}$ walleye pollock ${ }^{24}$ and southern blue whiting. ${ }^{26}$

All of the high-frequency models cited so far are similar in their neglect of diffraction. To remedy this, the boundaryelement method (BEM $)^{11}$ has begun to be applied in modeling scattering by swimbladder-bearing fish. ${ }^{14,15}$

In addition to treating diffraction, the BEM allows use of general conditions on the swimbladder boundary surface, with explicit representation of the internal fluid. Thus the BEM can also be used to study pressure-dependent effects, which are otherwise precluded by the standard Kirchhoffapproximation model. Establishing the validity of the BEM for a pressure-release surface is important for the larger program being introduced.

The present aim is to describe the two basic models that 
represent the swimbladder by its actual shape, namely the Kirchhoff-approximation model and the BEM, but both assuming a pressure-release boundary condition. Application of these to historical swimbladder morphometric data is described. Independent validation of the two methods is addressed, and computations with the two models are compared.

\section{KIRCHHOFF-APPROXIMATION MODEL}

In the Kirchhoff approximation, the field on the scattering surface is assumed to be known a priori. For a swimbladder-bearing fish at rather high frequencies, or wavelengths which are rather small compared to the maximum length of the swimbladder, the fish is represented by a pressure-release surface conforming to the inner wall of the swimbladder. ${ }^{23,24}$ The normal component of particle velocity on the scattering surface is assumed to be equal to that of the incident field on the directly insonified part of the surface, and zero on the geometrically shadowed part of the surface.

Mathematically, the farfield backscattering amplitude in this approximation is

$$
f=\lambda^{-1} \oint_{S} \exp (2 i \mathbf{k} \cdot \mathbf{r}) \mathcal{H}(\hat{\mathbf{k}} \cdot \hat{\mathbf{n}}) \hat{\mathbf{k}} \cdot \hat{\mathbf{n}} d S,
$$

where $\lambda$ is the acoustic wavelength, $\mathbf{k}$ is the wave vector in the source or backscattering direction $\hat{\mathbf{k}}=\mathbf{k} / k, \mathbf{r}$ is the position vector of the surface element with infinitesimal area $d S$, $\hat{\mathbf{n}}$ is the unit normal to $d S$ at $\mathbf{r}$, and $H(x)$ is the Heaviside step function with values 1 for $x>0, \frac{1}{2}$ for $x=0$, and 0 for $x<0$.

The integration in Eq. (1) is performed numerically using Gauss quadrature over curvilinear surface elements on which the position vector $\mathbf{r}$ is interpolated quadratically from nodal values. The integrand is evaluated at each integration, or Gauss, point using the interpolated value of r. A good representation of the phase, as given by the factor $\exp (2 i \mathbf{k} \cdot \mathbf{r})$, depends on the separation of these points. If the integration were to be performed by primitive Riemann summation, then the points should be closer than about $\lambda / 16$. However, the point-separation condition is undoubtedly relaxed by the use of Gauss quadrature. If the polynomial fit assumed by Gauss quadrature is of order 2 or higher, a good representation of the wave form should be obtained for a point separation up to $\lambda / 6$. For seven-point quadrature on six-node triangular elements, with nodes at the corners and midsides of the elements, the Gauss point separation is at most 0.8 times the nodal separation. A similar relationship exists for eight-node quadrilateral elements using $3 \times 3$ or more Gauss points. A condition for validity of the numerical integration, that the nodal separation should be less than $\lambda / 5$, is therefore tentatively suggested. This translates to a condition that the element side-to-wavelength ratio should be less than $2 / 5$. In order to give commonality with the corresponding condition in the case of the boundary-element method, to be discussed in Sec. III, the slightly stricter ratio of $1 / 3$ is adopted in this paper for assessing the frequency range of validity of a given mesh. The element meshes are described in Sec. IV.
The backscattering cross section is

$$
\sigma=4 \pi|f|^{2} \text {. }
$$

The target strength is the logarithmic expression of $\sigma$,

$$
\mathrm{TS}=10 \log \left[\frac{\sigma}{4 \pi r_{0}^{2}}\right],
$$

where $r_{0}$ is a reference distance, assumed here to be $1 \mathrm{~m}$.

\section{BOUNDARY-ELEMENT METHOD}

To develop the acoustic boundary-element method (BEM), the wave equation for the pressure $p$ is reduced to the Helmholtz form by assuming the harmonic time dependence $\exp (i \omega t)$, where $\omega$ is the angular frequency in radians per second, hence $\nabla^{2} p+k^{2} p=0$, where $k=\omega / c$ is the wave number. This is rewritten in integral form, in which the pressure at any point is expressed in terms of the acoustic pressure and normal displacement $u$ on the scattering surface $S$. This surface is subdivided into elements, and the pressure and displacement distributions on $S$ are represented by discrete values, $p_{i}$ and $u_{i}$, respectively, at each node $i$ associated with these elements. The standard Helmholtz integral equation suffers from singularities at certain critical frequencies, which are dense at high frequencies. To overcome this problem, the integral is combined with a second integral developed from the first by differentiating with respect to the normal direction at the surface. ${ }^{31}$ In principle, the two equations are combined by adding the standard form evaluated at each node of each element to a multiple $\alpha$ of the normalderivative form evaluated at the centroid of that element in the local coordinate system. ${ }^{11}$ The resulting equation can be written thus:

$$
\mathbf{A p}=\mathbf{B u}-\mathbf{p}_{\text {inc }}-\alpha \frac{\partial \mathbf{p}_{\text {inc }}}{\partial n} .
$$

If the swimbladder is assumed to be ideally pressurereleasing, $p_{i}=0$ for all $i$, and Eq. (4) can be solved directly for the nodal normal displacements:

$$
\mathbf{u}=\mathbf{B}^{-1}\left(\mathbf{p}_{\text {inc }}+\alpha \frac{\partial \mathbf{p}_{\text {inc }}}{\partial n}\right) .
$$

The coefficients of the matrix $\mathbf{B}$ are assembled from local matrices pertaining to each element of the mesh. With the calculation point of the Helmholtz integral taken at node $i$, with position $\mathbf{r}_{i}$, integration over element $m$ provides the following coefficients in the standard formulation:

$$
b_{m n}^{(1)}\left(\mathbf{r}_{i}\right)=\rho \omega^{2} \int_{S_{m}} N_{n}(\mathbf{q}) \cos \theta_{m n} G\left(\mathbf{r}_{i}, \mathbf{q}\right) d S_{q},
$$

where $\rho$ is the fluid density, $\mathbf{q}$ is the position vector of the integration point on the element surface $S_{m}, G$ is the Green's function, given by $G\left(\mathbf{r}_{i}, \mathbf{q}\right)=e^{-\mathrm{i} k\left|\mathbf{r}_{i}-\mathbf{q}\right|} / 4 \pi\left|\mathbf{r}_{i}-\mathbf{q}\right|, n$ is the local nodal label, and $N_{n}(\mathbf{q})(n=1,2, \ldots, 6$ for triangular elements, $n=1,2, \ldots, 8$ for quadrilateral elements) are the shape functions, which are of the standard second-order (quadratic) form. ${ }^{32}$ The factor $\cos \theta_{m n}$ is included to allow for the deviation $\theta_{m n}$ of the normal to the element $m$ at local node $n$ from the mean normal at that node. The mean normal at a node is 
TABLE I. Properties of the 15 specimens whose swimbladder surfaces have been remapped, based on the original mapping (Ref. 23 ) with $n_{\text {tri }}$ small triangular facets, by $n_{\text {elem }}$ curvilinear elements with $n_{\text {nodes }}$ nodes. The nodal separation distance such that $99 \%$ of neighboring separations are smaller and the maximum nodal separation are both specified.

\begin{tabular}{|c|c|c|c|c|c|c|c|c|c|c|}
\hline \multirow[b]{2}{*}{ Fish No. } & \multirow[b]{2}{*}{ Species } & \multirow[b]{2}{*}{$\begin{array}{l}\text { Length } \\
(\mathrm{cm})\end{array}$} & \multirow[b]{2}{*}{$\begin{array}{l}\text { Mass } \\
(\mathrm{g})\end{array}$} & \multirow[b]{2}{*}{$\begin{array}{c}\text { Ref. } 23 \\
n_{\text {tri }}\end{array}$} & \multicolumn{2}{|c|}{ New meshes } & \multicolumn{2}{|c|}{ Swimbladder } & \multicolumn{2}{|c|}{ Nodal separation } \\
\hline & & & & & $n_{\text {elem }}$ & $n_{\text {nodes }}$ & $\begin{array}{l}\text { Surface area } \\
\qquad\left(\mathrm{cm}^{2}\right)\end{array}$ & $\begin{array}{l}\text { Volume } \\
\left(\mathrm{cm}^{3}\right)\end{array}$ & $\begin{array}{l}99 \% \text { limit } \\
(\mathrm{mm})\end{array}$ & $\begin{array}{l}\text { Max } \\
(\mathrm{mm})\end{array}$ \\
\hline 201 & Pollack & 31.5 & 195 & 5546 & 1168 & 3364 & 33.01 & 6.91 & 1.20 & 2.21 \\
\hline 202 & Pollack & 44.0 & 533 & 9965 & 1389 & 4041 & 58.83 & 16.33 & 1.37 & 1.77 \\
\hline 204 & Pollack & 35.5 & 321 & 6562 & 1078 & 3116 & 42.39 & 10.03 & 1.41 & 1.72 \\
\hline 205 & Pollack & 39.0 & 380 & 7171 & 1107 & 3181 & 45.75 & 11.34 & 1.43 & 1.93 \\
\hline 206 & Pollack & 35.0 & 287 & 5379 & 1159 & 3347 & 31.37 & 7.75 & 1.17 & 1.46 \\
\hline 207 & Pollack & 44.5 & 635 & 8695 & 1487 & 4363 & 65.24 & 19.15 & 1.34 & 1.61 \\
\hline 209 & Saithe & 38.5 & 385 & 6762 & 1501 & 4387 & 43.29 & 10.08 & 1.06 & 1.39 \\
\hline 213 & Pollack & 34.5 & 259 & 10192 & 1039 & 2935 & 34.11 & 7.83 & 1.33 & 1.61 \\
\hline 214 & Pollack & 39.0 & 406 & 7649 & 1164 & 3362 & 44.14 & 10.15 & 1.34 & 1.53 \\
\hline 215 & Pollack & 37.0 & 332 & 5265 & 1076 & 3092 & 38.89 & 8.75 & 1.34 & 1.74 \\
\hline 216 & Pollack & 36.5 & 343 & 6436 & 1062 & 3060 & 43.33 & 10.85 & 1.40 & 1.64 \\
\hline 217 & Pollack & 34.5 & 253 & 5500 & 962 & 2764 & 34.61 & 7.11 & 1.32 & 1.46 \\
\hline 218 & Pollack & 32.5 & 257 & 4689 & 1327 & 3879 & 29.75 & 6.27 & 1.00 & 1.39 \\
\hline 219 & Pollack & 35.5 & 292 & 5106 & 1039 & 3005 & 35.74 & 8.15 & 1.27 & 1.53 \\
\hline 220 & Saithe & 38.0 & 406 & 8968 & 1321 & 3857 & 44.32 & 10.46 & 1.13 & 1.32 \\
\hline
\end{tabular}

defined as the average of the normals at the node on all contiguous elements weighted by the respective differential surface area.

The normal-derivative form of the Helmholtz integral equation, calculated at the centroids $\overline{\mathbf{r}}_{l}$ of the elements, similarly provides coefficients as follows:

$$
b_{m n}^{(2)}\left(\overline{\mathbf{r}}_{l}\right)=\rho \omega^{2} \int_{S_{m}} N_{n}(\mathbf{q}) \cos \theta_{m n} \frac{\partial G\left(\overline{\mathbf{r}}_{l}, \mathbf{q}\right)}{\partial n_{r}} d S_{q},
$$

where the normal derivative is evaluated at the centroid. These are combined with the previous coefficients by adding a multiple of $b_{m n}^{(2)}\left(\mathbf{r}_{l}\right)$ for all elements $l$ on which global node $i$ lies, i.e.,

$$
b_{m n}\left(\mathbf{r}_{i}\right)=b_{m n}^{(1)}\left(\mathbf{r}_{i}\right)+\alpha_{i} \sum_{l: i \varepsilon S_{l}} b_{m n}^{(2)}\left(\overline{\mathbf{r}}_{l}\right),
$$

where the combination factor $\alpha_{i}$ is taken to be $-\mathrm{i} / k M_{i}$, where $M_{i}$ is the number of elements meeting at node $i .^{11,33,34}$

The use of the centroids, rather than the nodes, as the calculation points for the normal-derivative form is found to be sufficient to overcome the problem of the critical frequencies while not increasing the computational effort unduly. ${ }^{11}$

The integrals are evaluated numerically using Gauss quadrature.

The coefficients $b_{m n}\left(\mathbf{r}_{i}\right)$ are assembled into the global matrix $\mathbf{B}$ by summing the coefficients that correspond to the same global node, thus

$$
B_{i j}=\sum_{m, n: C(m, n)=j} b_{m n}\left(\mathbf{r}_{i}\right),
$$

where $C(m, n)$ is the global node label of local node $n$ on element $m$. The source terms in Eq. (4) are evaluated thus:

$$
\mathbf{p}_{\text {inc }}\left(\mathbf{r}_{i}\right)+\alpha_{i} \sum_{l: i \varepsilon S} \frac{\partial \mathbf{p}_{\text {inc }}\left(\overline{\mathbf{r}}_{l}\right)}{\partial n_{r}}
$$

Given the solution for $\mathbf{u}$ from Eq. (5), the scattered pressure at any exterior point $\mathbf{r}$ is obtained from the standard integral equation by calculating coefficients similar to $b_{m n}^{(1)}\left(\mathbf{r}_{i}\right)$ but with $\mathbf{r}_{i}$ replaced by the position vector $\mathbf{r}$ :

$$
b_{j}^{(3)}(\mathbf{r})=\rho \omega^{2} \sum_{m, n: C(m, n)=j} \int_{S_{m}} N_{n}(\mathbf{q}) \cos \theta_{m n} G(\mathbf{r}, \mathbf{q}) d S_{q}
$$

and then

$$
p(\mathbf{r})=-\mathbf{b}^{(3)}(\mathbf{r}) \cdot \mathbf{u} .
$$

The backscattering amplitude at finite range $r$ is

$$
f(r)=\frac{r|p(\mathbf{r})|}{\left|p_{\text {ind }}\right|} .
$$

The farfield backscattering amplitude $f$ is the limit of $f(r)$ as $r$ approaches infinity. Expressions for the backscattering cross section and target strength are derived by substituting $f(r)$, or $f$, in Eqs. (2) and (3), respectively.

The elements used here are quadrilaterals and triangles of the quadratic isoparametric type, in which both the geometric and acoustic quantities are interpolated from the nodal values using quadratic shape functions, the nodes being situated at the vertices and midsides. ${ }^{32}$ As a general guide, good representation of the acoustic variables is obtained if the lengths of the sides of the elements are less than one-third of a wavelength. The accuracy of geometrical representation depends on the degree of undulation of the surface, but it should be noted that the quadratic interpolation allows the elements to be curved. Further details of the formulation and equations can be found in Ref. 11 .

\section{SWIMBLADDER MORPHOMETRY}

The origin of the morphometric data is a study performed in $1980^{35}$ on surface-adapted specimens of pollack and saithe, described briefly in Table I. Each specimen was anesthetized, tethered, and acoustically measured at each of four frequencies, nominally $38,50,70$, and $120 \mathrm{kHz}$, prior to shock-freezing and microtoming in the sagittal plane, hence 

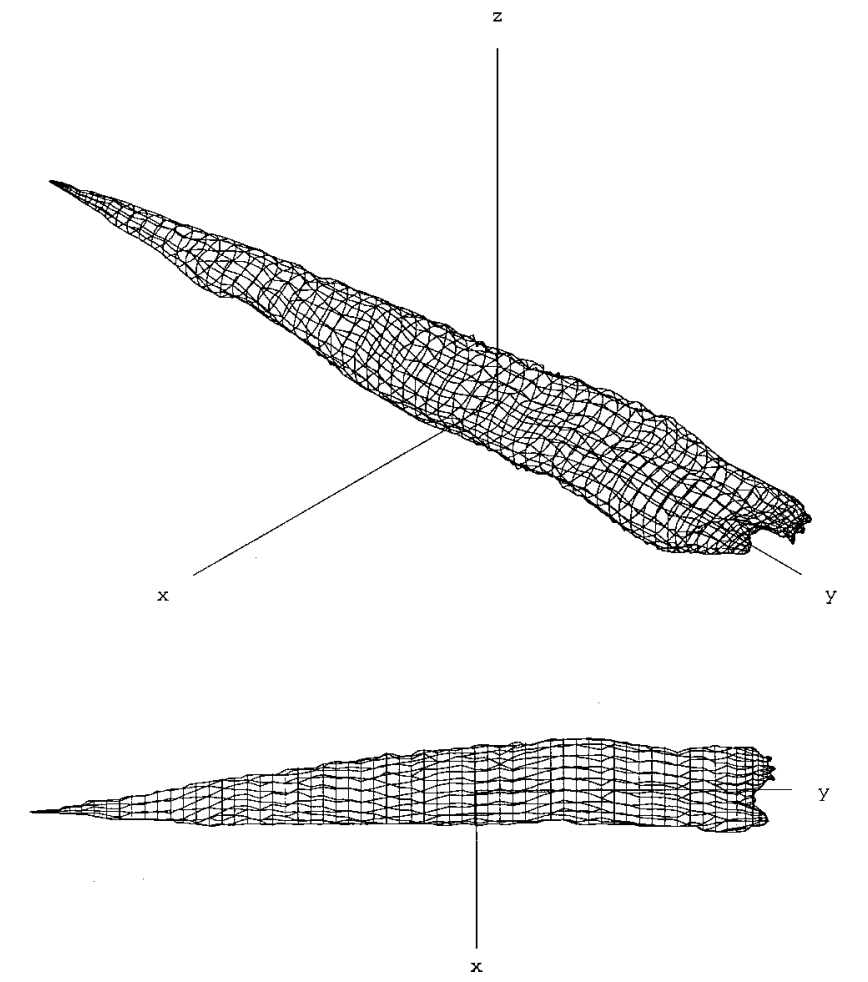

FIG. 1. Boundary-element mesh of the swimbladder to specimen No. 205 of Ref. 23, shown in both oblique and dorsal views. The model has 1107 elements and 3181 nodes. The meshed swimbladder length is $141 \mathrm{~mm}$.

parallel to the main axis, according to the method of Ona. ${ }^{36}$ The thickness of successive photographed sections was 100 $\mu \mathrm{m}$. Each swimbladder section was digitized as a set of coordinates describing the outline of the swimbladder, and the surface of each swimbladder was represented by a mesh consisting of flat triangular facets. ${ }^{23}$

Because of the use of curvilinear quadrilaterals and triangles in the BEM, new meshes have been produced for each of the specimens using a semiautomatic process. First, the contours of the swimbladder in planes perpendicular to the major axis of the fish, and hence perpendicular to the microtomed sections, are determined at intervals along the major axis, by finding the points of intersection of each plane with the original digitized sections. Quadrilateral and triangular elements are then fitted between neighboring contours. Where required, nodes are interpolated using cubic splines. This method allows the fineness of the mesh to be controlled by the choice of the separation between the contours and the nodal separation on each contour. Some manual fitting of elements is required where the swimbladder branches into separate lobes.

A further reason for the remapping exercise was to reduce the number of nodes in order to facilitate matrix operations inherent to the BEM. The resulting meshes have fewer elements than the original triangular meshes, ${ }^{23}$ but this is offset by the allowance for curvature of the surface. An example of one of the meshes is visualized in Fig. 1. The new meshes have been used in computations with the Kirchhoffapproximation model in parallel with the BEM.

Details of the meshes are listed in Table I. For each mesh, the maximum distance between neighboring nodes is shown in the final column, column 11; however, a better indication of the degree of fineness of each mesh is given in column 10, namely the limit of nodal spacing which is satisfied by $99 \%$ of the distances between pairs of neighboring nodes. At $120.4 \mathrm{kHz}$ the nodal spacing, to satisfy the $\lambda / 6$ condition for accuracy of the BEM and Kirchhoffapproximation model, should be less than $2.03 \mathrm{~mm}$. All of the meshes except that for specimen 201 are well within this limit even on the basis of the maximum nodal spacing found in the mesh. Detailed analysis of the mesh for specimen 201 reveals that the $\lambda / 6$ condition is satisfied for all but two pairs of neighboring nodes out of 4530 such pairs.

\section{INDEPENDENT VALIDATION OF MODELS}

A cogent form of validation of the Kirchhoffapproximation model is the direct comparison of model computations and measurement results for the same fish specimens used in the morphometry. This work is documented in detail in Ref. 23 but in which the integration in Eq. (1) is performed by the primitive Riemann summation, with evaluation of the integrand at the centroid of each triangular facet.

Validation of the BEM has already been documented for a series of cases in which analytical solutions are available. Three of those described in Ref. 11 are cited. (1) The forward scattered pressure for a plane wave incident on a rigid sphere has been computed. The agreement over the $k a$ range from 0 to 10 is excellent, without discrepancies at the critical frequencies that arise in the standard formulation, which lacks the normal component included in Eq. (4). (2) The backscattered pressure from a rigid sphere has been computed under similar conditions, again with excellent agreement, while avoiding discrepancies at the critical frequencies. (3) As an illustration, the radiation impedance of a uniformly vibrating circular piston of radius $a$ in the end face of a cylinder of radius $2 a$ and height $4 a$ over the $k a$ range from 0 to 5 has been computed and compared with the analytical solution for a piston in an infinite baffle. With allowance for the difference between the two problems, the agreement is quite good.

An additional trial of the BEM has been designed specifically for the present study. A spherical void of radius 25 $\mathrm{mm}$ is assumed to be immersed in sea water of sound speed $1470 \mathrm{~m} / \mathrm{s}$ and density $1025 \mathrm{~kg} / \mathrm{m}^{3}$. The size has been chosen for having an area of $7854 \mathrm{~mm}^{2}$, which is roughly $20 \%$ greater than the area of the mesh spanning the surface of the largest swimbladder, No. 207, as represented by 1487 elements (4363 nodes), with an area of $6524 \mathrm{~mm}^{2}$. Meshes have been generated by subdividing each spherical triangle of a geodesic icosahedron into four subtriangles, subdivided again to get a mesh of 320 elements (642 nodes) and subdivided once more to get a mesh of 1280 elements (2562 nodes). The latter mesh has a maximum nodal separation of $2.06 \mathrm{~mm}$, which is just outside the limit of $2.03 \mathrm{~mm}$ required by the $\lambda / 6$ condition at $120.4 \mathrm{kHz}$.

The same example provides a trial for the Kirchhoff approximation, since the integration in Eq. (1) can be performed analytically for the spherical shape: 


$$
\begin{aligned}
f= & (4 k)^{-1}\{-1+\cos (2 k a)+2 k a \sin (2 k a) \\
& +i[\sin (2 k a)-2 k a \cos (2 k a)]\},
\end{aligned}
$$

where $a$ is the sphere radius. Comparison of the numerical and closed-form analytic solutions at the frequencies 38.1, 49.6, 68.4, and $120.4 \mathrm{kHz}$ demonstrates agreement to within $0.01 \mathrm{~dB}$ for the 2562-node sphere. Further exercise of the Kirchhoff model reveals significant divergence of the numerical solution from the exact solution at about $180 \mathrm{kHz}$ for the 2562-node sphere and at about $90 \mathrm{kHz}$ for the 642-node sphere. The truth of this last statement is evident in the target strength spectrum in Fig. 2, which also compares the results of the Kirchhoff approximation with the exact series solution and BEM solution for the 642-node mesh. The observed deviation of the BEM solution from the exact series solution at about $90 \mathrm{kHz}$ corresponds to a nodal spacing of $\lambda / 4$, which is coarser than the nominal criterion for validity of the BEM, namely neighboring-point separations within $\lambda / 6$.

Numerical computations have been performed for both the BEM and Kirchhoff-approximation model for each of the two meshes. The computations have been repeated at each of the four measurement frequencies, 38.1, 49.6, 68.4, and $120.4 \mathrm{kHz}$, hence with $k a=4.07,5.30,7.31$, and 12.87. The results have been compared against the well-known analytical solution for scattering by a spherical void, with perfectly soft boundary condition, in a homogeneous fluid with given sound speed and density values. ${ }^{37}$ In one set of computations, the effect of orientation of the axis of the meshed void is examined by comparing the backscattered pressure amplitude at infinity for the Kirchhoff-approximation model and at 1000-m range for the BEM. The results are shown in Table II. In a second set of computations, the same backscattered pressure amplitude for a single orientation is compared directly against the amplitude derived with the analytical solution. The results of these computations, as well as those for the forward scattered amplitude by the analytical solution and BEM, are presented in Table III. Corresponding target strengths and forward-scattering strengths are presented in Table IV.

It is noted that, for certain frequencies and mesh sizes, the condition for the nodal spacing discussed in Secs. II and III, namely that this should be less than $\lambda / 6$, is violated. The results for these cases are included in Tables II-IV but are shown in italics. The limit is only just exceeded by the finer mesh at $120.4 \mathrm{kHz}$.

\section{COMPARISON OF MODEL COMPUTATIONS}

The target strength for an immersed void with the shape of the swimbladder shown in Fig. 1 has been computed as a function of tilt angle for both the dorsal and ventral aspects at each of four frequencies. Both the Kirchhoffapproximation model and BEM have been examined. The computational results are shown with the measured functions in Fig. 3.

The same computations have been repeated for the mapped swimbladder shapes of all 15 gadoid specimens listed in Table I. In order to reduce these to manageable proportions, the several functions have been averaged with respect to normal distributions $g(\theta)$ of tilt angle $\theta$, with mean $\bar{\theta}$ and standard deviation $s_{\theta}$ :

$$
\bar{\sigma}=\int g(\theta) \sigma(\theta) d \theta / \int g\left(\theta^{\prime}\right) d \theta^{\prime},
$$

where the integration has been performed over the range $[\bar{\theta}$ $\left.-3 s_{\theta}, \bar{\theta}+3 s_{\theta}\right]$. For the measured target strength functions, values at tilt angles outside the range $\left[-45^{\circ}, 45^{\circ}\right]$ were not available, and for such angles the value of $\sigma$ at the nearest angle limit has been used.

Computations have been performed for each of four normal distributions of tilt angle. The paired values $\left(\bar{\theta}, s_{\theta}\right)$ are $\left(0^{\circ}, 0^{\circ}\right),\left(0^{\circ}, 5^{\circ}\right),\left(0^{\circ}, 10^{\circ}\right)$, and $\left(-4.4^{\circ}, 16^{\circ}\right)$. Because of the effect of perspective, by which the apparent tilt angle of a fish changes as it is observed at different positions in the plane transverse to the acoustic axis, ${ }^{38}$ the effective values of $s_{\theta}$ are larger than the nominal ones. The values shown above have been adjusted for the perspectival effect for a circular beam of $5^{\circ}$ beamwidth measured between the half-power points. The effective standard deviations for the four cases are $2.5^{\circ}, 5.5^{\circ}, 10.2^{\circ}$, and $16^{\circ} . .^{23}$

The target strength corresponding to each averaged backscattering cross section, denoted $\overline{\mathrm{TS}}$, has been computed by substituting the value of $\bar{\sigma}$ from Eq. (14) in Eq. (3). The values of $\overline{\mathrm{TS}}$ have been regressed on fish length $l$ in centimeters according to the regression equation,

$$
\overline{\mathrm{TS}}=20 \log l+b,
$$

where the regression coefficient $b$ is expressed in decibels. The standard error of the regression has been computed for each derived regression equation. The results are shown in Tables V and VI.

\section{DISCUSSION}

\section{A. Model validation computations}

To validate the BEM for application to the gadoid swimbladder, a 25-mm-radius spherical void in sea water has been chosen as a test case in order to have a shape for which a rather simple analytical solution exists and whose surface area is greater than that of the largest swimbladder in the data set. Finite-element representation of the sphere by a subdivided icosahedron has allowed both the BEM and Kirchhoff approximation to be computed according to Eqs. (12) and (1), respectively.

Because of the finiteness of the facets, there is an effect due to axial orientation, which is indicated in Table II. The BEM is seen to be quite accurate for the two meshes that were chosen. Sensitivity to axial orientation is negligible as long as the maximal nodal separation does not exceed $\lambda / 6$. The Kirchhoff-approximation model is exercised with the identical meshes but performs less well than the BEM; it is inherently different, as is proved by the difference in respective exact and analytical solutions for the two models for this special shape. The variability with orientation is notable for the coarser mesh. 


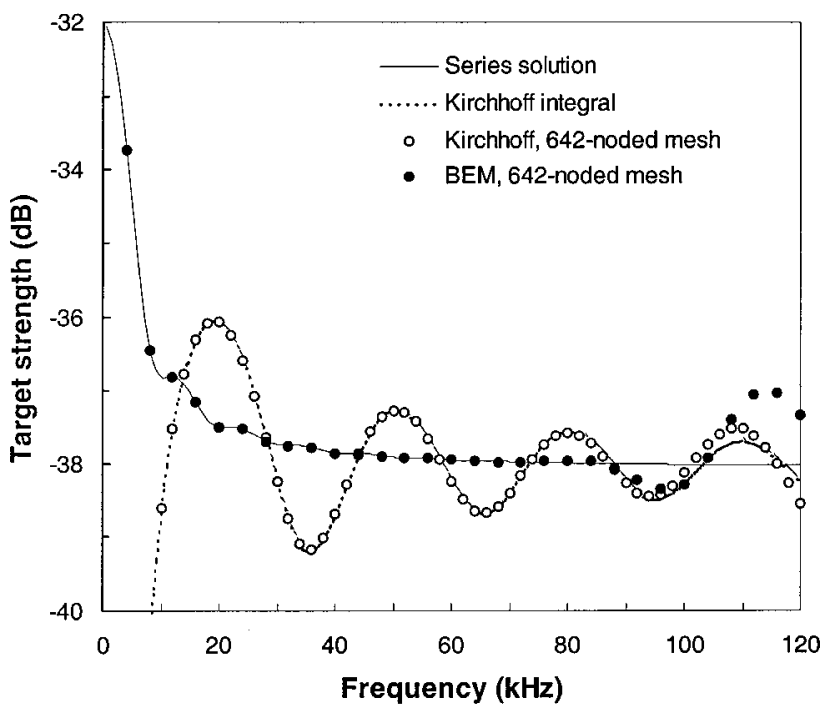

FIG. 2. Frequency dependence of a spherical void of radius $25 \mathrm{~mm}$ immersed in water of density $1025 \mathrm{~kg} / \mathrm{m}^{3}$ and sound speed $1470 \mathrm{~m} / \mathrm{s}$, as given by the series solution, Kirchhoff integral through the closed-form expression in Eq. (13), numerical Kirchhoff approximation using a surface mesh with 642 nodes, and boundary-element method using the same 642-node surface mesh.

Both backscattering and forward-scattered pressure amplitudes for the same boundary-element meshes are shown in Table III for the BEM, with close agreement with the analytical result except for the coarser mesh at the highest frequency of $120.4 \mathrm{kHz}$, where the nodal spacing greatly exceeds $\lambda / 6$. Again, the Kirchhoff approximation performs less well. Differences in the two models are also evident in the logarithmic measures presented in Table IV.

The apparent improvement in performance of the Kirchhoff approximation at the highest frequency of $120.4 \mathrm{kHz}$ is illusory, since, as noted previously, the closed-form analytic solution for the Kirchhoff-approximation model for the spherical void differs from the exact series solution. In fact, the approximation solution given by Eq. (13) oscillates about the analytical solution with a cycle frequency of about 30 $\mathrm{kHz}$, corresponding to the condition $2 \Delta k a=\pi$, where $\Delta k$ describes the change in wave number that satisfies the equality, as shown in Fig. 2. The corresponding Kirchhoffapproximation model results for the two meshes agree well with each other except at the highest frequency, where the separation in neighboring Gauss points for the coarser mesh, using seven-point integration, is about $\lambda / 4$, thus exceeding $\lambda / 6$. The nodal spacing for the same mesh is about $\lambda / 3$, also exceeding both the $\lambda / 5$ and $\lambda / 6$ conditions mentioned in Secs. II and III, respectively.

The Kirchhoff approximation, since it requires only a direct integration, is computationally much faster than the BEM, which requires the inversion of a complex matrix of size equal to the square of the number of nodes. Above about 4000 nodes, the BEM becomes cumbersome, at least on current desk-type workstation-level computers. In contrast, the Kirchhoff approximation could be exercised with far more elements than used here and thus, in principle, could be more amenable to computation at higher frequencies. However, the approximation has inherent limitations, such as the neglect of diffraction over the surface of the scattering shape or body, if most serious at relatively low frequencies.

To summarize, the BEM results agree well with the analytical results for the cases of 2562 and 642 nodes, deviating significantly only for nodal separations exceeding $\lambda / 6$. The Kirchhoff approximation fares relatively poorly in comparison; nonetheless, it agrees to within about $1.2 \mathrm{~dB}$ at $38.1 \mathrm{kHz}$ and within about $0.6 \mathrm{~dB}$ at the three highest frequencies.

\section{B. Swimbladder-shape-based computations}

The detailed computations of target strength as a function of tilt angle are shown for a single specimen, No. 205, in Fig. 3. Both the Kirchhoff-approximation model and BEM results are shown for the swimbladder as represented in Fig. 1. The respective measured target strength functions are also shown.

Both similarities and deviations are observed. Significantly for this work, the Kirchhoff-approximation and BEM results are quite similar. Comparisons against the measured function reveal similarities in overall structure, but with sizable discrepancies over certain angular regions, both in amplitude and degree of structure. Nonetheless, in the displayed case of specimen No. 205, it can be imagined that some average backscattering cross sections will be similar in magnitude.

Statistical combination of the individual averages in the regression analysis described by Eq. (15) reveals strong similarities, especially for the tilt angle distribution with largest

TABLE II. Backscattered pressure amplitude relative to the incident pressure amplitude in units of $1 \times 10^{-6}$ for a 50-mm-diam spherical void immersed in sea water, calculated at $1000 \mathrm{~m}$ range for the analytical seriesexpressed solution and BEM, and scaled to $1000 \mathrm{~m}$ for the farfield Kirchhoff approximation. The effect on the amplitude of the orientation of the subdivided icosahedron used to represent the void in the BEM and Kirchhoff approximation is expressed through the parenthetical quantity, which is the maximum percentage deviation from the mean observed over a wide range of axial orientations. Values in italics indicate that the model has a nodal separation exceeding $\lambda / 6$ at the specified frequency.

\begin{tabular}{lcccc}
\hline \hline Frequency $(\mathrm{kHz})$ & 38.1 & 49.6 & 68.4 & 120.4 \\
$k a$ & 4.071 & 5.300 & 7.309 & 12.866 \\
\hline Analytical solution & 12.850 & 12.721 & 12.634 & 12.545 \\
BEM, 2562 nodes & 12.846 & 12.716 & $12.630(0.1)$ & $12.541(0.3)$ \\
BEM, 642 nodes & $12.839(0.1)$ & $12.710(0.4)$ & $12.616(1.6)$ & $12.587(28.4)$ \\
Kirchhoff, 2562 nodes & $11.199(0.6)$ & $13.692(0.8)$ & $11.801(1.0)$ & $12.219(1.4)$ \\
Kirchhoff, 642 nodes & $11.187(2.5)$ & $13.707(3.7)$ & $11.784(4.8)$ & $12.060(29.1)$ \\
\hline \hline
\end{tabular}


TABLE III. Back- and forward-scattered pressure amplitudes relative to the incident pressure amplitude in units of $1 \times 10^{-6}$ for a 50 -mm-diam spherical void at 1000 -m range for the analytical series-expressed solution and BEM, and scaled to $1000 \mathrm{~m}$ for the farfield Kirchhoff approximation. A single, fixed orientation is assumed for the axis of the subdivided icosahedron used to represent the void in the BEM and Kirchhoff approximation. The percentage deviation relative to the analytical solution is given.

\begin{tabular}{|c|c|c|c|c|c|c|c|c|}
\hline \multirow[t]{2}{*}{ Frequency $(\mathrm{kHz})$} & \multicolumn{2}{|c|}{38.1} & \multicolumn{2}{|c|}{49.6} & \multicolumn{2}{|c|}{68.4} & \multicolumn{2}{|c|}{120.4} \\
\hline & Back & Forward & Back & Forward & Back & Forward & Back & Forward \\
\hline Analytical solution & 12.850 & 78.658 & 12.721 & 95.306 & 12.634 & 122.240 & 12.545 & 195.700 \\
\hline BEM, 2562 nodes & 12.847 & 78.644 & 12.716 & 95.290 & 12.629 & 122.225 & 12.542 & 195.695 \\
\hline Percentage error & -0.026 & -0.018 & -0.038 & -0.017 & -0.036 & -0.012 & -0.026 & -0.003 \\
\hline BEM, 642 nodes & 12.839 & 78.625 & 12.707 & 95.269 & 12.617 & 122.204 & 13.511 & 195.544 \\
\hline Percentage error & -0.088 & -0.042 & -0.106 & -0.039 & -0.135 & -0.030 & 7.696 & -0.080 \\
\hline Kirchhoff, 2562 nodes & 11.203 & & 13.686 & & 11.808 & & 12.228 & \\
\hline Percentage error & -12.817 & & 7.586 & & -6.538 & & -2.527 & \\
\hline Kirchhoff, 642 nodes & 11.206 & & 13.677 & & 11.820 & & 11.769 & \\
\hline Percentage error & -12.794 & & 7.515 & & -6.443 & & -6.186 & \\
\hline
\end{tabular}

standard deviation, $N\left(-4.4^{\circ}, 16^{\circ}\right)$. This distribution, in fact, is the first and possibly only one of a gadoid, cod (Gadus morhua), determined photographically in situ. ${ }^{39}$ In this case, in dorsal aspect, the Kirchhoff approximation agrees with measurement to within $0-1 \mathrm{~dB}$ over all four frequencies, while the BEM results differ by $0.7-1.5 \mathrm{~dB}$, as documented in Table V. For the tilt angle distribution with nominal standard deviation of $10^{\circ}$, the difference between the Kirchhoff and measured TS functions is $0.1-1.5 \mathrm{~dB}$. The difference between the BEM and measured TS functions is $1.2-2 \mathrm{~dB}$. For the narrower tilt angle distributions, differences are larger, reflecting differences in structure of the modeled backscattering amplitude at central angles.

For the ventral aspect, with results presented in Table VI, differences are smaller. The difference between Kirchhoff and measured results at the broadest tilt angle distribution is $0.7-1.9 \mathrm{~dB}$. For the BEM and measured results, the difference is $0.4-1.1 \mathrm{~dB}$.

The BEM and Kirchhoff-approximation results are quite similar except at the lowest frequency, $38.1 \mathrm{kHz}$, where the greatest discrepancy in regression coefficient is $1.3 \mathrm{~dB}$. At this frequency, the Kirchhoff-approximation result is closer to the result based on measurement in dorsal aspect for all four tilt angle distributions. In ventral aspect, the BEM result is closer.

At $49.6 \mathrm{kHz}$, the greatest difference in Kirchhoffapproximation and BEM results is $0.7 \mathrm{~dB}$, with average difference about $0.4 \mathrm{~dB}$. At $68.4 \mathrm{kHz}$, the greatest difference is
$0.4 \mathrm{~dB}$, with average difference about $0.2 \mathrm{~dB}$. At $120.4 \mathrm{kHz}$, the respective numbers are 0.8 and $0.5 \mathrm{~dB}$.

A survey of the overall results in Tables V and VI may suggest some systematic differences with the regression coefficient $b$ and standard error. Caution is advised in the interpretation of apparent systematic differences, however, because the computational results are not all independent. While 16 sets of computations are presented for each aspect, they apply to a total of only four frequencies, which is closer to the number of degrees of freedom for the respective aspect. Were data available at other frequencies, the possible presence of systematic differences among measurements and models might reveal the influence of other parts of the fish in modifying backscattering by the swimbladder alone.

A further internal comparison is possible for the Kirchhoff approximation. The mapping of the swimbladder is derived from the original mapping by flat triangular facets. ${ }^{23}$ The new mapping, for consistency with the BEM, contains fewer but curvilinear elements spanning the swimbladder surface. A detailed comparison of corresponding values for the regression coefficient $b$ in Eq. (15) reveals a greatest discrepancy of $0.2 \mathrm{~dB}$, with median discrepancy of $0.1 \mathrm{~dB}$, for the dorsal aspect. In ventral aspect the greatest discrepancy is $0.1 \mathrm{~dB}$, with median discrepancy less than $0.05 \mathrm{~dB}$.

\section{Summary of comparisons}

Earlier validation exercises with the BEM have been supplemented by a new example, that of a spherical void for

TABLE IV. Back- and forward-scattered pressure amplitudes shown in Table III, but expressed in decibels relative to 1-m range and incident wave amplitude.

\begin{tabular}{|c|c|c|c|c|c|c|c|c|}
\hline \multirow[t]{2}{*}{ Frequency $(\mathrm{kHz})$} & \multicolumn{2}{|c|}{38.1} & \multicolumn{2}{|c|}{49.6} & \multicolumn{2}{|c|}{68.4} & \multicolumn{2}{|c|}{120.4} \\
\hline & Back & Forward & Back & Forward & Back & Forward & Back & Forward \\
\hline Analytical solution & -37.822 & -22.085 & -37.910 & -20.418 & -37.969 & -18.256 & -38.031 & -14.168 \\
\hline BEM, 2562 nodes & -37.824 & -22.087 & -37.913 & -20.419 & -37.972 & -18.257 & -38.033 & -14.168 \\
\hline BEM, 642 nodes & -37.830 & -22.089 & -37.919 & -20.241 & -37.981 & -18.258 & -37.387 & -14.175 \\
\hline Kirchhoff, 2562 nodes & -39.013 & & -37.274 & & -38.556 & & -38.253 & \\
\hline Kirchhoff, 642 nodes & -39.011 & & -37.280 & & -38.547 & & -38.585 & \\
\hline
\end{tabular}



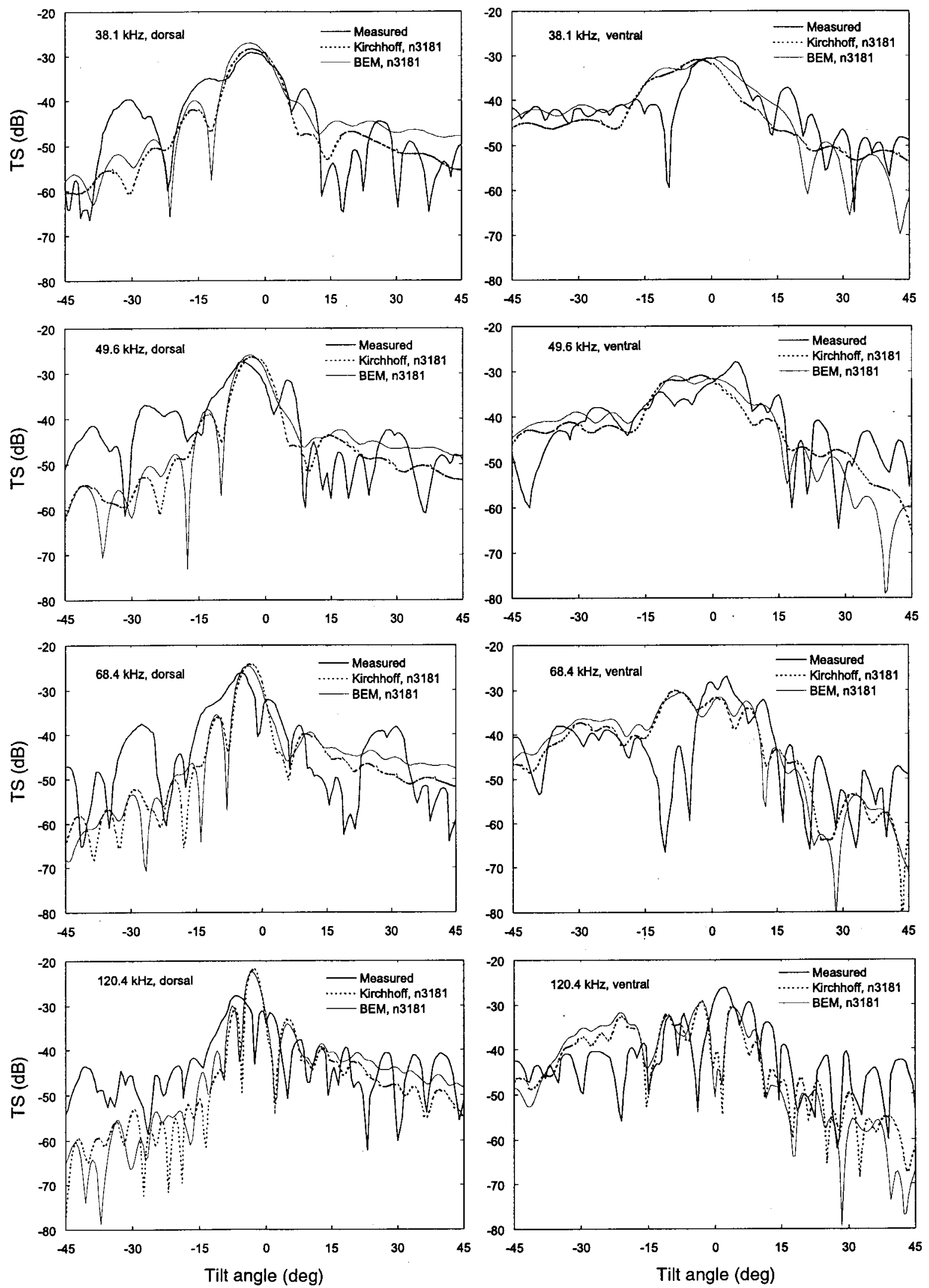

FIG. 3. BEM and Kirchhoff-approximation-model computations of target strength as a function of tilt angle compared against direct measurements for specimen No. 205. The functions are shown for both dorsal and ventral aspects at each of four frequencies.

which a simple analytical solution is known. Results with the Kirchhoff approximation are poorer. Nonetheless, in the case of the swimbladder-shape-based computations, the Kirchhoff approximation, when exercised with the curvilinear elements used in the BEM, yields results that agree well with earlier computations carried out using meshes with larger numbers 
TABLE V. Regression coefficient for the target strength-fish length relationship based on computations or measurements of each of 15 specimens in dorsal aspect when averaged over four distinct distributions of tilt angle $\theta$, characterized by the mean $\bar{\theta}$ and standard deviation $s_{\theta}$, abbreviated s.d. The coefficient $b$ is that shown in Eq. (15). The associated standard error of the regression, SE, is also shown.

\begin{tabular}{|c|c|c|c|c|c|c|c|c|}
\hline \multirow{2}{*}{$\begin{array}{l}\text { Frequency } \\
\qquad(\mathrm{kHz})\end{array}$} & \multicolumn{2}{|c|}{$\begin{array}{l}\text { Tilt angle distribution } \\
\text { (deg) }\end{array}$} & \multicolumn{2}{|c|}{ BEM } & \multicolumn{2}{|c|}{ Kirchhoff } & \multicolumn{2}{|c|}{$\begin{array}{l}\text { Measured TS } \\
\text { functions }\end{array}$} \\
\hline & Mean & s.d. & $b$ & SE & $b$ & SE & $b$ & $\mathrm{SE}$ \\
\hline 38.1 & 0.0 & 0.0 & -61.3 & 1.0 & -62.3 & 1.2 & -63.5 & 1.3 \\
\hline 38.1 & 0.0 & 5.0 & -62.3 & 0.6 & -63.6 & 0.9 & -64.3 & 0.6 \\
\hline 38.1 & 0.0 & 10.0 & -64.1 & 0.4 & -65.4 & 0.7 & -65.7 & 0.4 \\
\hline 38.1 & -4.4 & 16.0 & -65.7 & 0.4 & -67.0 & 0.6 & -66.9 & 0.4 \\
\hline 49.6 & 0.0 & 0.0 & -60.8 & 1.1 & -61.1 & 1.3 & -64.8 & 1.1 \\
\hline 49.6 & 0.0 & 5.0 & -61.9 & 0.6 & -62.4 & 0.8 & -64.1 & 0.7 \\
\hline 49.6 & 0.0 & 10.0 & -63.8 & 0.4 & -64.4 & 0.7 & -65.0 & 0.6 \\
\hline 49.6 & -4.4 & 16.0 & -65.4 & 0.4 & -66.1 & 0.6 & -66.1 & 0.6 \\
\hline 68.4 & 0.0 & 0.0 & -60.2 & 1.3 & -60.0 & 1.4 & -64.7 & 1.2 \\
\hline 68.4 & 0.0 & 5.0 & -61.4 & 0.7 & -61.5 & 0.8 & -63.9 & 1.0 \\
\hline 68.4 & 0.0 & 10.0 & -63.3 & 0.5 & -63.5 & 0.7 & -64.8 & 0.7 \\
\hline 68.4 & -4.4 & 16.0 & -65.0 & 0.5 & -65.2 & 0.6 & -65.9 & 0.6 \\
\hline 120.4 & 0.0 & 0.0 & -59.3 & 1.6 & -59.8 & 1.8 & -64.8 & 1.6 \\
\hline 120.4 & 0.0 & 5.0 & -60.7 & 1.2 & -61.2 & 1.3 & -63.8 & 1.3 \\
\hline 120.4 & 0.0 & 10.0 & -62.7 & 1.1 & -63.2 & 1.2 & -64.7 & 1.1 \\
\hline 120.4 & -4.4 & 16.0 & -64.4 & 1.0 & -64.9 & 1.2 & -65.9 & 0.9 \\
\hline
\end{tabular}

of flat elements.

While use of the BEM does not improve the earlier agreement of Kirchhoff-approximation computations with the measured target strength functions, ${ }^{23}$ the two models do show a basic agreement. Differences in predictions, as expressed through the regression coefficient in Eq. (15), are less than $1 \mathrm{~dB}$ in all cases except at $38.1 \mathrm{kHz}$ where the greatest difference is $1.3 \mathrm{~dB}$. There is some expectation that the discrepancy might be largest at the lowest frequency, for the Kirchhoff approximation assumes high frequencies. An offsetting factor, however, is the effective element side-towavelength ratio, which increases with frequency, decreasing the applicability, hence accuracy, of the approximation.

\section{Future work}

The BEM performs similarly to the Kirchhoff approximation for the frequencies and swimbladder sizes considered here. This is reassuring, since both models have the great

TABLE VI. Regression coefficient for the target strength-fish length relationship based on computations or measurements of each of 15 specimens in ventral aspect when averaged over four distinct distributions of tilt angle $\theta$, characterized by the mean $\bar{\theta}$ and standard deviation $s_{\theta}$, abbreviated s.d. The coefficient $b$ is that shown in Eq. (15). The associated standard error of the regression, SE, is also shown.

\begin{tabular}{|c|c|c|c|c|c|c|c|c|}
\hline \multirow{2}{*}{$\begin{array}{l}\text { Frequency } \\
(\mathrm{kHz})\end{array}$} & \multicolumn{2}{|c|}{$\begin{array}{l}\text { Tilt angle distribution } \\
(\text { (deg) }\end{array}$} & \multicolumn{2}{|c|}{ BEM } & \multicolumn{2}{|c|}{ Kirchhoff } & \multicolumn{2}{|c|}{ Measured } \\
\hline & Mean & s.d. & $b$ & SE & $b$ & $\mathrm{SE}$ & $b$ & SE \\
\hline 38.1 & 0.0 & 0.0 & -64.8 & 1.9 & -65.2 & 1.8 & -63.7 & 1.5 \\
\hline 38.1 & 0.0 & 5.0 & -65.0 & 1.2 & -65.7 & 1.2 & -64.3 & 0.9 \\
\hline 38.1 & 0.0 & 10.0 & -65.8 & 0.7 & -66.7 & 0.8 & -65.7 & 0.7 \\
\hline 38.1 & -4.4 & 16.0 & -66.7 & 0.4 & -67.8 & 0.7 & -67.1 & 0.7 \\
\hline 49.6 & 0.0 & 0.0 & -65.7 & 2.5 & -65.2 & 2.2 & -62.7 & 1.4 \\
\hline 49.6 & 0.0 & 5.0 & -65.4 & 1.4 & -65.3 & 1.4 & -63.4 & 0.8 \\
\hline 49.6 & 0.0 & 10.0 & -65.9 & 0.7 & -66.2 & 0.9 & -64.6 & 0.6 \\
\hline 49.6 & -4.4 & 16.0 & -66.7 & 0.5 & -67.2 & 0.7 & -66.0 & 0.6 \\
\hline 68.4 & 0.0 & 0.0 & -66.0 & 2.5 & -65.6 & 2.6 & -62.3 & 1.3 \\
\hline 68.4 & 0.0 & 5.0 & -65.7 & 1.5 & -65.5 & 1.7 & -63.0 & 0.7 \\
\hline 68.4 & 0.0 & 10.0 & -66.0 & 0.8 & -66.1 & 0.9 & -64.3 & 0.5 \\
\hline 68.4 & -4.4 & 16.0 & -66.6 & 0.6 & -67.0 & 0.8 & -65.8 & 0.5 \\
\hline 120.4 & 0.0 & 0.0 & -66.0 & 1.9 & -66.4 & 2.2 & -62.5 & 1.2 \\
\hline 120.4 & 0.0 & 5.0 & -65.6 & 1.2 & -66.0 & 1.4 & -62.8 & 0.7 \\
\hline 120.4 & 0.0 & 10.0 & -66.0 & 0.9 & -66.6 & 1.2 & -64.0 & 0.5 \\
\hline 120.4 & -4.4 & 16.0 & -66.6 & 0.9 & -67.4 & 1.2 & -65.5 & 0.5 \\
\hline
\end{tabular}


advantage over other models in being able to treat a complicated shape without compromise, in principle, based on a finite-element representation of the surface.

The BEM can, however, do much more than the Kirchhoff-approximation model. (1) It can, for example, treat lower frequencies, for diffraction is addressed in a fundamental manner through the integral-equation solution of the wave equation described in Sec. III. (2) The BEM can also treat the case of a gas-filled swimbladder under the influence of hydrostatic pressure without having to make assumptions about the applicable boundary conditions. (3) A third advantage of the model is its capacity to treat heterogeneous structures, as long as the heterogeneities can be demarked by surfaces within which the acoustic properties are uniform. Thus, the BEM can predict scattering by a swimbladderbearing fish in which the swimbladder and other structures, e.g., liver, reproductive products, and fish tissue surrounding the swimbladder, are explicitly represented. (4) It is noted that the BEM can address finite-scattering-range effects, without having to assume an infinite range, as is done in typical farfield-only models.

The applicability of the BEM to organisms other than swimbladder-bearing fish is evident. Thus the BEM can address scattering by the organisms conveniently classified by Stanton as deformed fluid cylinders, deformed elastic-shelled spheres, and bubble plus tissue, as represented, for example, by euphausiids, gastropods, and siphonophores, respectively. ${ }^{40}$ Significantly, there is no need to approximate shape or boundary conditions, for the model is numerical.

Some of the effects mentioned here may be addressed in a future work. These may also include bistatic scattering, which is allowed by both the BEM and Kirchhoffapproximation model, as well as other models, such as the deformed-cylinder model. ${ }^{10}$

\section{ACKNOWLEDGMENTS}

This work began with sponsorship by the European Commission through its RTD-program, Contract No. MAS3CT95-0031 (BASS). A Norton is thanked for much valued assistance in the technical editing. This is Woods Hole Oceanographic Institution Contribution No. 10437.

${ }^{1}$ R. H. Love, "Measurements of fish target strength: A review," Fish. Bull. 69, 703-715 (1971).

${ }^{2}$ L. Midttun, "Fish and other organisms as acoustic targets," Rapp. P.-V. Reun.-Cons. int. Explor. Mer. 184, 25-33 (1984).

${ }^{3}$ K. G. Foote, "Summary of methods for determining fish target strength at ultrasonic frequencies," ICES J. mar. Sci. 48, 211-217 (1991).

${ }^{4}$ S. McClatchie, G. Macaulay, R. F. Coombs, P. Grimes, and A. Hart, “Target strength of an oily deep-water fish, orange roughy (Hoplostethus atlanticus). I. Experiments," J. Acoust. Soc. Am. 106, 131-142 (1999).

${ }^{5}$ K. G. Foote, "Target strength of fish," in Encyclopedia of Acoustics, edited by M. J. Crocker (Wiley, New York, 1997), pp. 493-500.

${ }^{6}$ D. N. MacLennan, "Acoustical measurement of fish abundance," J. Acoust. Soc. Am. 87, 1-15 (1990).

${ }^{7}$ K. G. Foote, "Acoustic sampling volume," J. Acoust. Soc. Am. 90, 959964 (1991).

${ }^{8}$ J. B. Hedgepeth, V. F. Gallucci, F. O'Sullivan, and R. E. Thorne, “An expectation maximization and smoothing approach for indirect acoustic estimation of fish size and density," ICES J. mar. Sci. 56, 36-50 (1999).

${ }^{9}$ A. Stepnowski, and M. Moszinski, "Inverse problem solution techniques as applied to indirect in situ estimation of fish target strength," J. Acoust. Soc. Am. 107, 2554-2562 (2000).

${ }^{10}$ T. K. Stanton, "Sound scattering by cylinders of finite length. III. Deformed cylinders," J. Acoust. Soc. Am. 86, 691-705 (1989).

${ }^{11}$ D. T. I. Francis, "A gradient formulation of the Helmholtz integral equation for acoustic radiation and scattering," J. Acoust. Soc. Am. 93, 17001709 (1993).

${ }^{12}$ C. S. Clay and J. K. Horne, "Acoustic models of fish: The Atlantic cod (Gadus morhua)," J. Acoust. Soc. Am. 96, 1661-1668 (1994).

${ }^{13} \mathrm{~S}$. McClatchie, and Z. Ye, "Target strength of an oily deep-water fish, orange roughy (Hoplostethus atlanticus). II. Modeling," J. Acoust. Soc. Am. 107, 1280-1285 (2000).

${ }^{14}$ D. T. I. Francis and K. G. Foote, "Boundary-element-model predictions of acoustic scattering by swimbladder-bearing fish," Proceedings of the Fourth European Conference on Underwater Acoustics, edited by A. Alippi and G. B. Cannelli (CNR-IDAC, Rome, Italy, 1998), Vol. I, pp. 255260.

${ }^{15}$ K. G. Foote and D. T. I. Francis, "Target strength spectra of swimbladdered fish," J. Acoust. Soc. Am. 105, 1111 (1999).

${ }^{16}$ R. I. C. C. Francis and M. R. Clark, "Inferring spawning migrations of orange roughy (Hoplostethus atlanticus) from spawning ogives,"Mar. Freshwater Res. 49, 103-108 (1998).

${ }^{17}$ I. Andreeva, "Scattering of sound by air bladders of fish in deep sound scattering ocean layers," Sov. Phys. Acoust. 10, 17-20 (1964).

${ }^{18}$ D. E. Weston, "Sound propagation in the presence of bladder fish," in Underwater Acoustics, edited by V. M. Albers (Plenum, New York, 1967), Vol. 2, pp. 55-88.

${ }^{19}$ R. H. Love, "Resonant acoustic scattering by swimbladder-bearing fish," J. Acoust. Soc. Am. 64, 571-580 (1978).

${ }^{20} \mathrm{M}$. Furusawa, "Prolate spheroidal models for predicting general trends of fish target strength," J. Acoust. Soc. Jpn. (E) 9, 13-24 (1988).

${ }^{21}$ L. Ding and Z. Ye, "A method for acoustic scattering by slender bodies. II. Comparison with laboratory measurements." J. Acoust. Soc. Am. 102, 1977-1981 (1997).

${ }^{22}$ K. Sawada, Z. Ye, R. Kieser, G. A. McFarlane, Y. Miyanohana, and M. Furusawa, "Target strength measurements and modeling of walleye pollock and Pacific hake," Fish. Sci. 65, 193-205 (1999).

${ }^{23}$ K. G. Foote, "Rather-high-frequency sound scattering by swimbladdered fish," J. Acoust. Soc. Am. 78, 688-700 (1985).

${ }^{24}$ K. G. Foote and J. J. Traynor, "Comparison of walleye pollock target strength estimates determined from in situ measurements and calculations based on swimbladder form," J. Acoust. Soc. Am. 83, 9-17 (1988).

${ }^{25}$ S. McClatchie, J. Alsop, Z. Ye, and R. F. Coombs, "Consequence of swimbladder model choice and fish orientation to target strength of three New Zealand fish species," ICES J. mar. Sci. 53, 847-862 (1996).

${ }^{26}$ S. McClatchie, G. Macaulay, S. Hanchet, and R. F. Coombs, "Target strength of southern blue whiting (Micromesistius australis) using swimbladder modelling, split beam and deconvolution," ICES J. mar. Sci. 55, 482-493 (1998)

${ }^{27}$ T. K. Stanton, "Sound scattering by cylinders of finite length. I. Fluid cylinders," J. Acoust. Soc. Am. 83, 55-63 (1988).

${ }^{28}$ T. K. Stanton, "Sound scattering by cylinders of finite length. II. Elastic cylinders," J. Acoust. Soc. Am. 83, 64-67 (1988).

${ }^{29}$ T. K. Stanton, "Sound scattering by spherical and elongated shelled bodies," J. Acoust. Soc. Am. 88, 1619-1633 (1990).

${ }^{30}$ C. Partridge and E. R. Smith, "Acoustic scattering from bodies: Range of validity of the deformed cylinder method," J. Acoust. Soc. Am. 97, 784795 (1995).

${ }^{31}$ A. J. Burton and G. F. Miller, "The application of integral equation methods to the numerical solutions of some exterior boundary problems," Proc. R. Soc. London, Ser. A 323, 201-210 (1971).

${ }^{32}$ O. C. Zienkiewicz and R. L. Taylor, The Finite Element Method, 4th ed. (McGraw-Hill, London, 1989), Vol. 1, pp. 121-132.

${ }^{33}$ W. L. Meyer, W. A. Bell, M. P. Stallybrass, and B. T. Zinn, "Prediction of the sound field radiated from axisymmetric surfaces," J. Acoust. Soc. Am. 65, 631-638 (1979).

${ }^{34} \mathrm{~T}$. Terai, "On calculation of sound fields around three dimensional objects by integral equation methods," J. Sound Vib. 69, 71-100 (1980).

${ }^{35} \mathrm{~K}$. G. Foote, "Linearity of fisheries acoustics, with addition theorems," J. Acoust. Soc. Am. 73, 1932-1940 (1983).

${ }^{36}$ E. Ona, "Physiological factors causing natural variations in acoustic target strength of fish," J. Mar. Biol. Assoc. U.K. 70, 107-127 (1990).

${ }^{37}$ R. R. Goodman and R. Stern, "Reflection and transmission of sound by elastic spherical shells,” J. Acoust. Soc. Am. 34, 338-344 (1962). 
${ }^{38}$ K. G. Foote, "Averaging of fish target strength functions," J. Acoust. Soc. Am. 67, 504-515 (1980).

${ }^{39} \mathrm{~K}$. Olsen, "Orientation measurements of cod in Lofoten obtained from underwater photography, and their relation to target strength," Council
Meeting of the International Council on Explor. Sea 1971/B, 17, 8 pp.

${ }^{40}$ T. K. Stanton, "Simple approximate formulas for backscattering of sound by spherical and elongated objects," J. Acoust. Soc. Am. 86, 1499-1510 (1989). 